Ludovico FULCI

Università di Wrocław

\title{
MACHIAVELLI MODERNO O POSTMODERNO?
}

\section{Premessa}

Che il pensiero di Machiavelli resista imperterrito all'usura del tempo e che anzi abbia fin qui accompagnato grandi trasformazioni compiutesi nel corso dell'età moderna è una cosa che è sotto gli occhi di tutti.

La Rivoluzione francese ne rivitalizzò le teorie politiche ${ }^{1}$, creando l'immagine di un Machiavelli amico del popolo che si sofferma a illustrare le nefandezze dei principi per l'utile dei sudditi che queste cose devono pure sapere. Per noi che ci interroghiamo sulla modernità è importante il fatto che Machiavelli non venisse considerato da chi si apprestava a varcare la soglia di quella che oggi chiamiamo l'età contemporanea un pensatore dell'Ancien Régime. L'aver oltrepassato indenne quel primo confine induce a chiedersi se, oltre che moderno, egli possa dirsi anche post-moderno.

Fuori d'Italia Machiavelli è comunque oggi uno degli autori italiani più letti e ricercati e si può affermare che il Principe è stato tradotto più volte quasi in tutte le lingue. La questione se Machiavelli possa costituire un elemento di continuità tra moderno e post-moderno si pone quindi legittimamente, specie considerando che con Machiavelli si è all'inizio di un percorso che tende a valorizzare la razionalità, ma di essa non ha fatto un valore. Su questo punto vanno, secondo noi, osservate alcune cose. La prima è che la razionalità è uno degli aspetti cruciali di una tradizione moderna di cui, proiettandosi in un'ipotetica dimensione di postmodernità, si cerca di stabilire un profilo (LYOTARD, $1987: 73)^{2}$. In secondo luogo ricordiamo come, nel nome di Machiavelli, si sia più volte sostenuta l'importanza di considerare l'imponderabile, cioè quel che sfugge alle previsioni ma che agisce all'interno della storia e che alcuni notano, altri no. Infine osserviamo che con Machiavelli si è all'inizio del delinearsi di un progetto, quello appunto della modernità, che sarebbe ingenuo ritenere trasmessosi nei secoli senza venire in qualche modo deformato. In questo senso il ritorno a Machiavelli ci sembra opportuno per chi, guardando al futuro, si ponga la questione di rintracciare il senso di un progetto morale, intellettuale, culturale forse ancora non del tutto attuato. In fondo la modernità, con la sua contraddittoria nostalgia dell'antico e l'impossibilità di mettere in parentesi il Medioevo che come età storica essa ha « inventato », consiste assai probabilmente in una congiuntura storica, una fase durata a lungo, in vista della realizzazione di un mondo diverso.

\footnotetext{
${ }^{1}$ Tale operazione si deve essenzialmente a Denis Diderot, considerato autore o ispiratore della voce « Machiavélisme » dell'Encyclopédie, nella quale si riprende la tesi spinoziana del Principe come satira del potere tirannico. Gli fece eco Jean-Jacques Rousseau che in un celebre passo del Contratto sociale lasciò scritto: "En feignant de donner des leçons aux Rois, il a donné de grandes aux peuples. Le Prince de Machiavel est le livre des républicains » (Contrat social, III,6).

${ }^{2}$ Ricordiamo che Lyotard prende le distanze da una tradizione di pensiero che si basa su un « racconto speculativo » o su un «racconto emancipativo», vale a dire su una tradizione che si trasmette raccontando l'avventura dell'intelletto o quelle di uno spirito che man mano si libera da vecchi legami.
} 


\section{Quando il duca Valentino incontrò messer Niccolò Machiavelli fiorentino}

$\mathrm{Ci}$ sono incontri che si manifestano come fatali. Altri che lo diventano, nel senso che per tali si chiariscono nel corso della vita. Un grande amore si riconosce abbastanza presto come incontro fatale e quelli leggendari di Dante con Beatrice e di Petrarca con Laura sono poi tanto credibili da essere stati assimilati nella credenza comune a fatti autentici e storicamente veri. L'incontro con una persona comunque eccezionale, e che avrebbe, alla luce di fatti successivi, potuto cambiare il corso naturale degli eventi dando una svolta sostanziale al corso della storia ivi compresa la nostra personale, è sicuramente un fatto memorabile nella vita di ciascuno di noi. Hegel - stando alla nota espressione contenuta in una sua lettera da lui scritta il giorno dopo aver terminato di scrivere la Fenomenologia dello Spirito - conservò sempre una viva impressione della maestà di Napoleone anima del mondo a cavallo e Francesco De Sanctis (1963 : 61) descrisse in modo commosso la visita di Leopardi alla scuola di Basilio Puoti, del quale De Sanctis era discepolo.

Ci pare ora che l'incontro di Machiavelli con Cesare Borgia sia stato recepito dalla letteratura critica e dalla tradizione interpretativa del machiavellismo come fatale in questo secondo senso, per il quale Machiavelli avrebbe intuito la grandezza di un personaggio destinato a grandi imprese. Quindi, riflettendo sulle vicende storiche che in quegli anni venivano dipanandosi immediatamente dopo il suo incontro col Valentino, Machiavelli avrebbe sempre meglio messo a fuoco la grandezza del personaggio, fino a farne il modello del Principe.

Non intendiamo capovolgere il giudizio e pretendere di riscrivere la storia di questo incontro. Crediamo anche noi a una folgorazione o infatuazione, chiaramente tutta letteraria e intellettuale, del Segretario della Repubblica fiorentina per quel che il Valentino mostrava di poter rappresentare alla luce di eventi politici successivi al loro incontro e alla parabola stessa dell'astro dei Borgia. Non pensiamo però che l'onesta catalogazione degli incontri "fatali" possa limitarsi alle due tipologie descritte - l'incontro obiettivamente memorabile e l'incontro che irretisce e affascina.

L'incontro, per esser tale, ha una sua reciprocità, e, se il lettore fa presto a intendere che il Machiavelli percepì quasi immediatamente la statura politica e morale del personaggio Cesare Borgia, resta pure aperta una questione a cui vorremmo in qualche modo rispondere: che ne è dell'incontro del Valentino con Machiavelli?

\section{L’incontro fra il duca e messer Niccolò}

Se il duca ha una posizione sociale di tutto rispetto, anche Machiavelli ha tutta l'apparenza di una persona alquanto fuori dell'ordinario e già questo legittimerebbe a parlare dell'incontro di Cesare Borgia con Niccolò Machiavelli. In fondo, che ne fossero coscienti o meno, è vero che l'astro politico del Valentino, destinato in breve a oscurarsi, è stato vivificato agli occhi della posterità soprattutto dall'attenzione che su di esso richiamò il Segretario della Repubblica fiorentina. Questo punto ci pare fondamentale ai fini dell'analisi che 
conduciamo circa la «fatalità » dell'incontro fra Cesare Borgia e Niccolò Machiavelli. Noi concediamo senza difficoltà che quest'ultimo sia, con la sua opera e in particolare con il Principe, un punto nodale della vicenda storica e culturale dell'Occidente, tanto da rappresentare la coscienza, come dicevamo, di quell'epoca. Ma è anche indubbio, agli occhi dei suoi contemporanei e perfino ai suoi stessi occhi, come di tale coscienza fosse campione piuttosto il Valentino, ovvero chiunque dall'osservatorio di un mondo in cui si pensa e si fa politica sia legittimato a plasmare la realtà storica, imprimendo ad essa quel carattere che per pregiudizio umanistico (sia pure corretto, ma non per questo corrotto, dalla spiritualità romantico-idealistica dei suoi tempi) Jacob Burckardt (1973) riferì all'uomo politico dell'età rinascimentale, creatore di quella forma di opera d'arte che sarebbe a suo dire lo stato ${ }^{3}$.

Insomma quella coscienza, che sarà da Hegel in poi legata a un pregiudizio intellettualistico che non ha diritto di cittadinanza nell'età rinascimentale, fa sì che non l'intellettuale ma il politico fosse nel Cinquecento, oltre che il protagonista della scena, anche la mente che intuisce, filtra, elabora, crea la storia. Una «mente» che, fuori del campo dell'azione reale e fattuale, elabori tattiche e strategie da seguire nelle scelte politiche è in qualche modo esclusa dallo stesso Machiavelli. Ė Machiavelli ad asserire che esistono tre generazioni, vale a dire tipi, di cervelli: "l'uno intende da sé, l'altro discerne quello che altri intende, el terzo non intende né sé né altri; quel primo è eccellentissimo, el secondo eccellente, el terzo inutile » (Il Principe, XXII, «De' secretari ch'e' Principi hanno appresso di loro $», \S 2)$.

Si può pensare che tale distinzione ricalcasse quella reale del maestro con due allievi, uno dei quali brillante e l'altro pigro. Non possiamo però ignorare il fatto che nella storia d'Europa, da un certo momento in poi, il maestro, un tempo maestro di vita, si sia trasformato in quel tipo di studioso che, mai stanco d'apprendere, si dedica alla riflessione e, osservando e ascoltando, impara. In questo senso si dovrà ammettere che la prima forma di intelligenza che Machiavelli concepisce è, ai suoi tempi, quella del politico, alla quale si affacciano le soluzioni dei problemi con un'immediatezza assoluta. La seconda forma è quella del filosofo, cioè dell'uomo di studi, che deve sulle cose riflettere, deducendo le verità da quanto altri gli dimostra essere evidente. La terza è il giudizio volgare che è politicamente irrilevante.

$\mathrm{Si}$ rifletta su questa distinzione, quale si pone in Machiavelli, e si comprenderanno meglio le ragioni della singolare fortuna del suo pensiero politico.

Se esaminiamo attentamente un tale giudizio, scopriamo che la mente politica, in quanto più risolutiva, è anche più irriflessiva di quella che intende la verità che le è da altri comunicata; per cui insomma la dote del politico è piuttosto nell'intuito, nel coraggio, nella prontezza, nella destrezza, laddove l'intellettuale, come studioso, comprende per via di un'attenzione posta riflessivamente sui fatti che esamina in modo distaccato, quando essi si sono già compiuti ovvero quando altri, nel compierli, lo sollecita a comprendere quel che da solo non comprenderebbe. Il politico $f a$ avendo intelligenza delle cose, delle situazioni, dei

\footnotetext{
${ }^{3}$ La prima parte dell'opera reca appunto il titolo di « Lo stato come opera d'arte ».
} 
fatti; l'intellettuale, il sapiente, il filosofo pensa, cioè soppesa, valuta i fatti (le res gestae) da altri portati a termine, con una prudenza che non è virtù politica. Del resto, se non gli difettano necessariamente, neanche necessariamente gli appartengono le due virtù dell'astuzia e del coraggio che Machiavelli riferisce al politico, cioè al principe.

E' ovvio peraltro che, quando si ragiona sulla base di categorie astratte, si rischia di generalizzare e insomma non esiste politico che, in quella realtà fattuale sulla quale Machiavelli ci ha insegnato a soffermare la nostra attenzione non assuma occasionalmente veste di filosofo e non esiste filosofo che non assuma occasionalmente veste di politico. Perciò, chiarendo il nostro pensiero, crediamo di poter asserire che anche agli occhi di Machiavelli la vera intelligenza è sempre comunque intelligenza politica, quindi prontezza che mette in condizione di interagire con i fatti, invece che subirli, secondo una visione che da sempre si riconosce al Rinascimento.

E qui arriviamo a un punto fondamentale. Per l'uomo dell'età rinascimentale il politico non è chi si uniforma alle direttive di un geniale consigliere e ancor meno è chi si attiene alle istruzioni di un ideologo. La tempestività dell'azione è requisito fondamentale dell'agire politico che per questo appare dettato da oscure necessità o da provvidenziali illuminazioni. Non parliamo solo di come si caratterizzasse l'arte del politico alla coscienza popolare, ci riferiamo a quell'imponderabile, a quel margine di incertezza di cui lo stesso politico è consapevole nell'età rinascimentale. $\mathrm{Ci}$ pare che in questo senso si debbano intendere molte delle raccomandazioni di Carlo V al figlio Filippo II, allorché questi si preparò a succedere al padre nel governo della Spagna.

\section{Machiavelli dopo Machiavelli}

E’ peraltro con Machiavelli che il ruolo del pensatore politico si definisce nei fatti, rivendicandosi uno spazio di riflessione autonomo intorno agli avvenimenti politici; spazio che spesso è momento di verifica, interpretazione, critica, che, in forma di giudizio pacato o di esaltazione e celebrazione, si fa in margine all'operato di coloro i quali ricoprono incarichi politici. Ne risulta un'implicita professione di modestia, probabilmente più sincera di quanto per pregiudizio comune non appaia, nel caso in cui lo scrittore molto conceda al tono elogiativo dell'encomio, in vita e post mortem tributato al personaggio eccezionale alla cui ombra egli sembra porre innanzitutto sé stesso ${ }^{4}$. Ma tale professione di modestia è poi dubbia nel caso dello storico che voglia entrare nei meccanismi più segreti, indagare nelle stanze del potere, secondo una prospettiva che, comunque individuata, non esclude l'intuizione politica di una possibile sopravvalutazione che a questo ruolo, naturalmente secondario, possono tuttavia dare alcune situazioni storiche nuove. Machiavelli è il primo intellettuale a dialogare a distanza ravvicinata con gli uomini di potere e a tratti si direbbe che avesse piena consapevolezza del privilegio che gli proveniva dal conoscere i meccanismi necessari a condurre le proprie analisi, a formalizzarle, a divulgarle.

\footnotetext{
${ }^{4}$ Si pensi ad esempio alla fortuna degli Elogi funebri di Bossuet, il quale aveva avuto, non a caso, l'incarico di educare il Delfino di Francia.
} 
Non può tacersi a questo punto che la fortuna politica dell'uomo di intelletto che si interroga sulle ragioni della grandezza del saggio uomo di governo, cresce sensibilmente proprio a cominciare dall'epoca di Machiavelli. Probabilmente in larga parte ciò si deve al fatto che il Rinascimento è l'epoca in cui nasce quello che Herbert Marshall McLuhan (1998) chiama fin nel titolo di una sua celebre opera l'uomo tipografico, il quale compie la sua educazione attraverso la lettura di classici, ai quali facendo riferimento può stabilire una rete di contatti con gli intellettuali europei. Non a caso, nessun movimento di idee risulta meglio gestito del Rinascimento, politicamente sorretto da opportuni chiarimenti programmatici in base ai quali si segue un iter educativo che dà a chi lo compie la consapevolezza di un qualche diritto da rivendicare. Inoltre è credibile che l'esperienza condotta a contatto dell'ambiente di corte consentisse all'umanista di accedere ai meccanismi di regia dell'apparato di corte. In tal modo egli comprende finalmente la politicità insita in fatti privi di rapporto immediato con quella che volgarmente è la politica, vale a dire la somma delle preoccupazioni del principe. Alludiamo ai filtri di cui il signore si avvale nel rapporto con i sudditi e che vanno dagli uffici della Cancelleria, alle parate; dalle feste carnascialesche alla messa in scena degli spettacoli; dall'opera che commissiona all'artista al poema che celebra i fasti del principe stesso e del suo casato. Questo aspetto della realtà è indagato a tutto obiettivo dall'umanista che gioca in tutte queste operazioni un ruolo fondamentale. Tutto questo in fondo è il Rinascimento, di cui alcune opere, singolarmente brevi e penetranti, come per esempio L'elogio della follia di Erasmo e il Principe, costituiscono quelli che oggi si chiamerebbero dei manifesti. In fondo il Rinascimento è quel che si è rigenerato ogni volta che la modernità in esso implicita è stata nuovamente invocata come momento essenziale alla crescita della società civile. Quest'ultima ha di fatto riconosciuto in opere divulgate, conservate, tramandate dall'invenzione della stampa, i suoi fondamentali monumenti.

\section{Machiavelli oltre il suo tempo}

Se dunque è vero che il Valentino agisce e Machiavelli pensa, è vero anche che il pensare di Machiavelli è un agire, un essere presente, un condizionare e orientare l'opinione altrui. Qui è, secondo noi, il primo seme della post-modernità del machiavellismo, che, col procedere della storia moderna sembra costantemente rinnovarsi, trovando come per miracolo nuove energie. Rispetto al pubblico che legge, almeno a partire dal Settecento ad oggi, è infatti Machiavelli ad aver inteso da sé, dove coloro che lo intendono nella lettura sono quegli eccellenti ai quali egli si rivolge. Ciò significa che egli ha sempre cose da dire, non tace mai, anzi si sovrappone ai fatti che racconta. Fin qui è riuscito a mettere in ombra, come abbiamo visto, l'intelligenza diretta che delle cose ha il principe, l'unico che sia tenuto a conoscere veramente $i$ fatti che egli gestisce per il ruolo politico che svolge e che, per pregiudizio generalmente condiviso dalla pubblica opinione dell'età di Machiavelli, gli spetta per diritto di nascita, un diritto naturale. Sono dunque i fatti storici posteriori a creare una frontalità nel rapporto tra $\mathrm{i}$ due grandi del pensiero politico rinascimentale: Machiavelli e il duca Valentino. 
Ma poi la storia procede. Si legge Machiavelli, ma si legge anche Bacon, nasce il metodo sperimentale e l'intellettuale pensa a un mondo da rifarsi a tavolino, che non nasca dalla capricciosa volontà di un potente, ma dalla ragionata disamina dei filosofi. Sarà Rousseau a portare bene in superficie, enfatizzandola, l'importanza che la voce dell'intellettuale ha, contrapponendo la statura morale di Machiavelli, censore dei potenti, con la prosa di un potere spietatamente esercitato ${ }^{5}$.

E' ovvio come una tale interpretazione, che da Diderot e Rousseau giunge, come ricordavamo, all'Ottocento italiano per la divulgazione che ne fece Ugo Foscolo, forzi la veridicità storica e che insomma Machiavelli non intendesse sfrondare gli allori del principe per svelare le lacrime e il sangue che grondano dietro l'edificante facciata di un potere che irretisce con fasti e grandi apparati scenografici ${ }^{6}$. Eppure che una contrapposizione di ruoli tra principe e umanista fosse nelle cose ci pare dovesse col tempo emergere. Quando l'umanista trova nel libro che scrive lo strumento nuovo per un nuovo potere e si rivolge al pubblico, egli occupa lo spazio fisicamente invisibile di una comunicazione che nella sua capillarità e forza di convincimento ha, nel tempo, effetti maggiori di quella che il principe esercita direttamente col fascino della propria persona, con la sua autorità, col suo carisma. Sicché l'interpretazione che Rousseau dà dell'opera di Machiavelli, fondata più che sul Principe anche e soprattutto sui Discorsi, ci pare all'origine di quella ragionevolezza che, come idealità morale, ha portato alla realizzazione di un modello di democrazia fondato sul parlamentarismo. Volendo anzi esaminare eventuali ricadute che a distanza di tempo il machiavellismo ha avuto negli spazi della politica italiana, non va sottovalutato il fatto che Machiavelli fosse nel primo Novecento una bandiera per un antifascismo che nella dittatura vedeva la rivalsa del principe contro l'umanista. Le parate, i discorsi, i filmati, i bagni di folla che la società di massa conosce, restituiscono all'uomo di potere tutto il fascino del divo pateticamente riducendo quello dell'intellettuale che ridiventa topo di biblioteca, professore, uomo d'altri tempi. Vi reagirà, in Italia, Alberto Moravia nel descrivere la trasformazione del personaggio di Quadri, che nel Conformista passa da un antifascismo teorico a uno militante. Si converrà che il personaggio di Quadri è appunto quello dell'intellettuale italiano (probabilmente concepito da Moravia sul modello dei suoi cugini Rosselli ${ }^{7}$ ) che sugli scritti di Machiavelli e di altri ha formato la sua coscienza politica, sapendosi all'occorrenza trasformare in uomo d'azione. Ed è questo un pregiudizio postilluministico e romantico, che affida all'intellettuale il compito di illuminare le menti dei potenti e la coscienza dei popoli. Presunzione su cui forse Machiavelli avrebbe sorriso.

Come dicevamo, infatti, volendo contestualizzare e rapportarsi alla realtà autentica d'un'epoca sacrificando prospettive interpretative suggerite da fatti e categorie mentali di là da venire, la questione di una rivalità, intesa in senso

\footnotetext{
${ }^{5}$ Cfr. nota 1 .

${ }^{6} \mathrm{Ci}$ riferiamo a un celebre passo dei Sepolcri in cui, alludendo a Machiavelli, Foscolo ne parla come di « quel grande / che temprando lo scettro a' regnatori, / gli allor ne sfronda ed alle genti svela / di che lacrime grondi e di che sangue » (vv. 135-138)

${ }^{7}$ E' quanto può dedursi da una dichiarazione dello stesso Moravia in un libro-intervista di Enzo Siciliano. Alla domanda circa i fatti storici che «stanno dietro » al Conformista lo scrittore risponde testualmente: "Il delitto Rosselli. In più voleva amalgamarvi quello che avevo conosciuto del fascismo ». (Cfr. Siciliano : 89).
} 
«alto », come competizione che non esclude emulazione, tra il Valentino e Machiavelli è cosa che non ha storia e, a rigore, non ha neanche ragione di porsi. Per meglio dire essa si pone a posteriori, da parte dello storico (cioè del professore di storia) il quale però, se compie un minimo sforzo per intendere il rapporto dialettico tra il politico e l'intellettuale dell'età rinascimentale, scopre che nessuna posizione è più privilegiata di quella del Valentino. Il fasto, la ricchezza, le relazioni di amicizia e di parentela di cui gode il duca nulla hanno a che vedere con la pur rilevante eredità di un nobile casato quale quello del fiorentino Machiavelli, che fuor di Firenze ha pure un nome. Tuttavia questo nome non suona alto quanto quello del figlio del papa, protetto del re di Francia, marito di una principessa di sangue reale e imparentato con gli Estensi. Se sul piano delle astratte elucubrazioni, la questione si pone più o meno legittimamente, sul piano di una percezione immediata e dunque più canonicamente vera (e, come vedevamo, politica) il gioco non si individua neppure. Va anzi aggiunto che l'età di Machiavelli è quella che prepara l'equiparazione in Europa della toga alla spada, ma la toga che per necessità riveste le spalle del nobile fiorentino è ancora, nel passaggio tra Quattrocento e Cinquecento, un segno di decadenza, come anche Ariosto esplicitamente lamenta circa l'ufficio di servitore del Cardinale d'Este che era stato costretto ad accettare per ristorare le finanze familiari.

Non bisogna tuttavia dimenticare che le politiche sono nell'Italia del Rinascimento almeno due: quella del principe e l'altra, più discreta, ma anche tanto più avveduta e prudente quanto più avverte la natura servile della propria funzione: quella del ministro (il termine significava in origine servitore), del consigliere, dell'esecutore, a cui nessuno ancora riconosce nel Rinascimento il ruolo di vero e proprio animatore della vita politica. In questo senso il principe continua ad essere l'eroe e dunque colui che dalla fortuna, vale a dire dalla condizione di nascita, dal capriccio degli eventi è stato chiamato a compiere le gesta che lo storico si prende cura di raccontare e testimoniare. La figura per così dire mediana del servitore, che da gentiluomo di camera, paladino, amministratore si avvia a conquistare un ruolo decisamente più politico (che è appunto quello del moderno ministro) tuttavia si prepara, senza forse una precisa consapevolezza, proprio con Machiavelli. Machiavelli che non fu consigliere, al quale anzi si chiuse la carriera politica all'indomani della restaurazione medicea, può legittimamente esser visto quale maestro dell'arte sottile di consigliare, di amministrare con prudenza all'ombra del potere e delle sue arroganze quella res publica che costituisce sicuramente l'idealità più generosa del repubblicano Machiavelli.

E sarà proprio la figura del ministro, del consigliere che assiste il principe, ad assorbire in sé quelle prerogative così tipiche dell' uomo politico. Il ministro avrà il sopravvento sul principe, per cui l'Europa moderna riconosce in Richelieu, in Mazarino, in Tayllerand, in Cavour e in Bismarck i campioni di una raffinata arte politica, prima ancora che in teste coronate in alcuni casi addirittura manovrate da questi grandi statisti. Si pensi alla rivalità tra Bismarck e Guglielmo, alla diffidenza di Vittorio Emanuele III verso Giovanni Giolitti, al modo in cui la regina Vittoria si destreggiò tra Palmerston e Disraeli.

Machiavelli, quale Segretario della Repubblica Fiorentina, e sia pur mancato leader politico, è l'antesignano dei fasti di un'azione che, per quanto meditata, è 
dettata da una sincera e profonda passione e da quella irresistibile ambizione che impone a coloro che la possiedono di gettarsi nella lotta, anzi nella «carriera » politica.

\section{Machiavelli fiorentino}

Se riflettiamo intorno a quella vera e propria damnatio memoriae di cui per secoli è stato oggetto il Principe, scopriamo che tutto ciò è legato indissolubilmente al bisogno da parte del politico di professione (il consigliere, il ministro della real casa) di formarsi a una letteratura che lo ponesse in condizione di svolgere il compito di tutore del principe, compito che grado a grado ottiene, promuovendosi di rango nelle mansioni politiche che è chiamato a svolgere. Il Principe mette un po' troppo a nudo verità che è meglio celare, di fronte al rischio di un oscuramento della dignità regale che, con la Rivoluzione Francese, perderà ogni attributo di naturale grandezza. Non siamo del resto i primi a insinuare che l'attività di storico e storiografo sia da considerarsi nel caso di Machiavelli un esercizio all'ombra di quella passione politica che nutrì la vita dell'austero repubblicano. E' nei saloni di Palazzo Vecchio che Machiavelli aveva individuato lo spazio per la discussione dei problemi relativi all'amministrazione di uno stato le cui dimensioni reali non superavano di molto i confini del comune fiorentino e che pertanto salvava la logica di un confronto « democratico » come oggi diciamo, e fondato sulla tutela delle libertà municipali e che allora erano artatamente ridotte ai maggiorenti della città, così a Firenze come in altri comuni italiani.

Non devono perciò trarre in inganno certi salamelecchi ed effusioni del tutto rituali, con cui il Segretario della Repubblica Fiorentina si rivolge al duca Valentino. Rientrano negli obblighi sociali e Machiavelli mancherebbe di tatto e di intelligenza nel sottrarsi a un dovere di buona creanza. La stima che peraltro ha del personaggio politico non ha nulla a che fare con i cerimoniali d'obbligo. Ma poi è da chiedersi: l'insistere sul Valentino, su quel che avrebbe potuto realizzare e tuttavia non ha realizzato per un complesso di circostanze fortuite; il tornare a parlarne anche nel contesto di quella che resta la sua opera maggiore, il Principe, tutto ciò ha solo il significato di un omaggio? E che senso mai avrebbe un tale omaggio a un defunto, ridondante, eccessivo nel suo zelo cavalleresco, in un'opera così agile, schematica, veloce ed essenziale quale è appunto il Principe? Vale inoltre la pena secondo noi interrogarsi più a fondo circa il significato di un'opera che, dedicata al Signore di Firenze, gli ricorda, sia pure di sfuggita, la grandezza di un personaggio che aveva macchiato le proprie mani del sangue di qualche suo parente: gli Orsini che il Valentino aveva mandato a morte erano cugini della moglie di Lorenzo il Magnifico, nonno del destinatario ufficiale dell'opera scritta da Machiavelli, e proprio il Magnifico aveva sposato Clarice Orsini, figlia di una sorella del cardinale Latino Orsini, padre di Paolo. Queste cose erano allora a tutti note in Italia (MACHIAVELLI, $2001: 20-23$ ).

Non ci sentiamo di escludere da parte dello scrittore una qualche consapevolezza d'essere stato anche lui attore, sia pure del tutto casualmente, di fatti memorabili. Osserviamo il documento da lui redatto che è poi la prima fonte in certo modo autobiografica del suo incontro con il Valentino. La celebre Descrizione del modo tenuto dal duca Valentino nello ammazzare Vitellozzo 
Vitelli, Oliverotto da Fermo, il signor Pagolo e il duca di Gravina Orsini fu frutto di rimaneggiamenti successivi (ibidem : 35), quasi che nel racconto degli avvenimenti di quei giorni ci fosse qualcosa di insolito che l'autore scopre con sorpresa in sé oltre che nei fatti di cui è testimone.

Il duca, per come la sua figura è in quell'opera tratteggiata da Machiavelli, è un personaggio che nel suo cammino travolge le persone che incontra. Ha l'affabilità cortese del principe quando al principe è consentito essere affabile in quanto deve dirimere questioni diplomatiche di grande interesse per sé e per i suoi progetti. E se qualcuno gli è di ostacolo, con molta freddezza il Valentino lo castiga. Machiavelli è per lui poco più che un nome. Eppure con messer Machiavelli conversa e ne sollecita, secondo quanto Machiavelli stesso riferisce, il parere. Segno di grande considerazione, ma anche di una certa volontà di gioco che un avversario forte si prende su chi sa essere meno agguerrito.

Sicuramente dunque l'incontro del Valentino con Machiavelli non è tale che ne possa immediatamente scaturire qualche valutazione o particolare apprezzamento da parte del Valentino o di altri sull'opera svolta dal personaggio inviato dalla Repubblica Fiorentina ad accogliere e fermare il duca, che non assalga Firenze, la quale è praticamente indifesa, almeno sul piano militare. Il compito di Machiavelli è reso più facile ma anche più delicato, vista la protezione che sulla città del Fiore ha posto il re di Francia Luigi XII col veto, che anche il Valentino non può ignorare, di assalirla. Si tratta di saggiare da un lato l'intelligenza, dall'altro la sete di potere del Valentino, che potrebbe anche solo differire il progetto di assalire Firenze. Anche su Machiavelli grava l'incertezza della capricciosa fortuna. Che cosa accadrebbe se Luigi XII dovesse all'improvviso venir meno? Quale freno interverrebbe a scongiurare un assedio del Valentino all'indifesa patria di Machiavelli?

Se si valutano le ragioni che avrebbe avuto il duca di scagliarsi su Firenze, si comprendono le legittime preoccupazioni del Segretario della Repubblica Fiorentina. Presa Firenze, il duca si sarebbe impossessato di una posizione strategica di sicuro valore, senza considerare le risorse che avrebbe potuto impiegare per condurre a termine la sua campagna militare nel resto d'Italia.

Per poco che si rifletta sugli strumenti culturali di cui Machiavelli dispone per tener testa a un interlocutore che potrebbe decidere di essergli nemico, non si può neanche lontanamente concludere che egli si avvalesse di altro che della sua personale sagacia, del suo spirito e di un sapere di vita.

Non vogliamo fare di Machiavelli il campione di ragioni che l'età moderna si è, in quanto moderna, voluta lasciare alle spalle. A noi non sfugge che lo stesso baconianesimo, visto come punto di partenza di una mentalità moderna e scientifica, in quanto sperimentale e razionale, si radica anch'esso in una cultura pre-scientifica $^{8}$. Pensiamo però che Machiavelli fosse figlio di una cultura in cui l'educazione del Principe è arte sottile, fatta di sapienza segreta che consiste nel riconoscere, come vedevamo, l'eccezionalità di una condizione di nascita e nello spingere una particolare natura, segnata da un innegabile privilegio della sorte, a manifestare nel modo più compiuto l'ineluttabilità di un destino guidato da una

\footnotetext{
${ }^{8}$ Circa l'eredità della magia nel pensiero di Bacone, Gassendi e Cartesio, fa considerazioni assai interessanti Paolo Rossi (Rossi Paolo, Francesco Bacone. Dalla magia alla scienza, Einaudi, Torino, 1964, pp. 17-52).
} 
stella favorevole. Nell'età di Machiavelli abbondano quegli astrologi di corte, dei quali Guicciardini dice: «Quanto sono più felici gli astrologi degli altri uomini! Quelli, dicendo tra cento bugie una verità, acquistano fede in modo che è creduto loro el falso; questi, dicendo tra molte verità una bugia, la perdono in modo che non è più creduto loro el vero » (I ricordi, aforisma 57).

\section{Machiavelli e la scienza alchemica}

Probabilmente anche messer Machiavelli avrebbe sorriso divertito a questa insinuazione che noi leggiamo col disincanto dei moderni pronti a cogliere l'aspetto paradossale di una verità tipica di quei tempi, dimenticando quanto il discrimine tra vero e falso sia stato per secoli, proprio nell'età moderna, terreno di insidie per il filosofo che, fino all'Ottocento aveva perso di vista l'importanza anche politica della fattualità di vero e falso. Vero e falso costituiscono una dicotomia innanzitutto alchemica e poi logica. E' il saggio della lega di metallo a consentire di distinguere la moneta vera da quella falsa. Si aggiunga poi che la falsità e l'inganno sono l'ossessione di tutta una cultura che per secoli aveva demandato a prove tanto cruciali quanto illogiche (si pensi all'ordalia) il compito di stabilire la verità. Nulla di strano quindi se anche a corte si pensa all'interrogazione sistematica delle stelle del cielo e se, secondo l'uso imposto dalle norme dell'araldica, si vuole che il capitano vittorioso si fregi di un'insegna in campo azzurro, concedendosi allo sconfitto, che sia sconfitto con onore, il campo rosso, secondo un contrasto di colori che qualsiasi regista teatrale prende ancor oggi in considerazione quando deve porre in scena un lavoro shakespeariano.

Sono dettagli apparentemente del tutto secondari, ma noi siamo invece convinti della loro importanza, dal momento che trasmettono in modo immediato informazioni circa quel che si prepara, quel che, non essendo ancora avvenuto, tuttavia avverrà. E' la psicologia, non l'astrologia a suggerirci che in fondo al suo cuore il torero sa già se verrà sconfitto o se vincerà un'altra volta ancora il toro. Chi ne sappia osservare i gesti, fin dal momento in cui entra nell'arena, può comprendere quale sarà il finale della lotta dell'uomo con l'animale e sulla base di neanche troppo vaghe e insulse suggestioni poetiche di cui sono esempio mille componimenti della tradizione classica, ritrovare nello scenario di un pomeriggio $i$ segni di una morte ineluttabile. Che è quanto fece Federico Garcia Lorca nel celebre Lamento per la morte di Ignazio. Né il fatto che oggi le cose siano totalmente mutate, per cui insomma si riesce a far sì che generalmente il toro venga sconfitto, ha per il nostro discorso qualche importanza. Non è solo la corrida ad aver perso il suo carattere di spettacolo rituale e perciò anche in qualche modo sacrale. La spettacolarità, con la gratuità di inutili sacrifici, è tipica del mondo d'oggi, che attenua l'aspetto cruento di certe ritualità, riuscendo quasi a insinuare l'idea che tutto sia finto, quanto più è poveramente e desolatamente vero.

Tornando però al Cinquecento, ci pare chiaro che il destino fosse allora da cercare in un quadro di conoscenze che un po' troppo sbrigativamente sono state nei tempi successivi destituite di fondamento e che invece erano operative all'epoca di Machiavelli. Alludiamo in breve a una cultura i cui statuti 
fondamentali non sono ancora quelli della scienza sperimentale, ma piuttosto quelli di un "sapere " che, per essere rapportato, ricondotto e misurato secondo l'eccellenza della persona che compie le sue esperienze, ha un carattere magico. Tale carattere non può machiavellicamente farsi consistere nel palese irretimento di coloro che, mancando di giudizio, cedono alle pratiche superstiziose e si lasciano convincere che certi apparati di cui il mago si avvale siano qualcosa di più che non semplici esteriorità. In altri termini ci interroghiamo sul senso della cultura magica, non della magia volgarmente intesa e riteniamo che la scienza politica di cui Machiavelli ragiona non sia altro che la magia, spogliata dei suoi inutili e fantasiosi orpelli.

Le astuzie meschine con cui il furbo irretisce l'ingenuo non caratterizzano il principe di Machiavelli che, sebbene abbia i suoi vizi, tuttavia ha sempre una qualche magnanimità, anche quando colpisce il nemico senza perdonarlo. Quel che in Machiavelli fa da contraltare alla fortuna è, non a caso, la virtù. Di questo termine, secondo noi, si è sottolineata un po' troppo l'ascendenza dotta, latina, dimenticandosi che nell'età medievale la virtus, come è intesa dai vari Lullo e dagli alchimisti è qualità intrinseca della cosa, facoltà che serve a descrivere la cosa in un suo tratto costitutivo e caratteristico. Secondo noi aveva pienamente ragione Oreste Tommasini (1883-1911, II : 39-41) nel sostenere che «né altro indica la parola virtù del politico fiorentino che quello che Galeno e i medici solevano designare col nome di virtus. Chi questo non ravvisa - proseguiva enfaticamente Tommasini - rischia di prendere ogni maniera di abbagli nel giudicare il sistema filosofico di lui. Chi negli umori del corpo sociale, di cui egli osserva a ogni piè sospinto, le aggregazioni, le alterazioni, le purgazioni, non riconosce analogie e allusioni frequenti e palesi ad aforismi galenici, non giungerà facilmente a rendersi esatta ragione della genesi dei pensieri di esso ».

La cultura medica, che è filosofica oltre che magica, è viva ancora per tutto il Rinascimento. Quanto all'uso del termine virtù nel senso detto, si pensi anche alla virtù d'erbe o d'arte maga di cui parla Petrarca (« I begli occhi ond'i' fui percosso in guisa / ch'e' medesmi porian saldar la piaga, / et non già vertú d'erbe, o d'arte maga » (Canzoniere, 75). Riferita all'uomo la virtù non è dunque, machiavellicamente, eroismo e, se aveva ragione Luigi Russo (1972 : 17) a sostenere che in Machiavelli la virtù è virtù individuale, bisogna anche ricordare che tale virtù non è la virtù morale che appartiene all'uomo "virtuoso », ma la forza della natura che opera nell'uomo e che l'uomo deve saper ben governare. «La nozione di virtù - suggerisce Donato Romano - compendia risorse diverse, che, riunite, di norma attraggono la fortuna: tra esse, la capacità di interpretare uomini e situazioni, l'ingegno creativo, la razionalità, il coraggio, il realismo, la prontezza. In politica chi è irresoluto finisce travolto dagli eventi, mentre chi ha virtù sa prendere decisioni tempestive, mutando rotta e atteggiamenti non appena le circostanze lo richiedano » (Machiavelli e la fondazione della scienza politica, in http://www.donatoromano.it/appunti/machiavelli.htm)

Il principio etico (o, se si preferisce, metaetico) della filosofia politica di Machiavelli è qui: nella convinzione che la condizione di nascita, i privilegi conseguenti a fatti casuali, la buona stella che favorisce i pochissimi che giungono al governo dei principati son cose che devono trovare nella virtù dell'uomo, cioè nel coraggio, nella forza del carattere e nell'intelligenza, uno strumento che 
assecondi il favore del destino, senza di che possibilmente la fortuna non arride, poichè la persona non è neanche in grado di vedere l'occasione che gli è offerta $\mathrm{e}$, continuando a disperare, vive la vita anonima e piatta di chi deve ignorare certe opportunità. Insomma, se togliamo la palla di vetro, l'interrogazione dei tarocchi, l'uso delle pietre e gli apparati esteriori dell'astrologia medievale, l'umanista Niccolò Machiavelli, che illustra ai principi le basi dell'arte della politica, è un indovino che, scrutando il comportamento del suo interlocutore, lo pone saggiamente a rinvenire in fatti pretestuosamente esterni, difficoltà personali e lo aiuta a vedere con maggiore chiarezza nell'orizzonte della propria vita. E' quanto sembra almeno in parte osservare Quintin Skinner (1999: 47 ), riferendosi a una lettera di Machiavelli a Giovanni Soderini nella quale si esprime la valutazione che « se ognuno sapesse comandare la propria natura, verrebbe ad esser vero che il savio può comandare alle stelle e ai fati »

Né questo è tutto. Infatti, a fronte di una funzione per così dire terapeutica dell'interrogazione delle stelle e delle carte, lo stesso gioco del mago svela di avere nelle pieghe più nascoste qualche plausibilità. Il suo interrogare le stelle circa il destino di un interlocutore che non sia uno sciocco credulone è assai simile al dialogo che l'uomo d'oggi ha con se stesso sul lettino dello psicanalista. La relazione che comunque costruisco tra i simboli rivenuti nei miei sogni e le figure che si succedono nelle mani del cartomante, è sforzo di una fantasia tesa a stabilire una connessione tra me e il mondo che mi circonda. Le storie di tradimenti, fedeltà, guerra e pace che un bambino spontaneamente inventa guardando un mazzo di carte, sono un gioco che nasconde questo bisogno con cui egli esercita un controllo sulle proprie capacità di gestire la sua realtà. Ora, comunque si voglia definire la politica - arte del compromesso, spregiudicata e sottile abilità nel piegare ai propri scopi la volontà degli altri - è sicuro che l'immaginario, insieme con la fantasia e l'invenzione, vi ha una parte predominante. La scienza politica che con Machiavelli nasce, non ha preso mai la strada dello sperimentalismo puro. Si pensi a questo riguardo a Max Weber che nel leader vede giustamente un capo carismatico, un personaggio che incute rispetto in quanto sa dominare le situazioni: nei termini del machiavellismo possiede una virtù che gli consente di conoscere e valutare rapidamente le situazioni, cercando di strappare la benda che nasconde lo sguardo della dea fortuna. Egli è arbitro della sua sorte, faber fortunae sui, secondo un motto latino tradizionalmente invocato per definire i termini di una cultura rinascimentale in cui l'uomo si fa protagonista della propria esistenza. Ciò significa che si intende aprire, per così dire, la metafora del cielo stellato, dei segni sulla mano, delle carte che estraiamo a caso. Tutte queste cose fingono la nostra esperienza, aristotelicamente intesa quale presa di contatto con la realtà, che, in quanto è tecnica, è manualità, anzi manipolazione.

Non si deve a nostro avviso sottovalutare l'attaccamento della cultura del tempo a un tale pregiudizio fondato sulla convinzione che il sapiente sia colui che ha conoscenza diretta delle cose. Non esiste ancora il laboratorio, non esistono strumenti di rilevazione e misurazione, l'osservazione è diretta, vale l'intuito. L'esperienza è sensata in quanto tale la rendono le capacità personali del saggio. Sarà Galilei a dover concedere quasi cent'anni dopo, che per intendersi meglio, occorre « fare un po' di disegno » (Dialogo sopra i massimi sistemi dell'universo, giornata III, parla Simplicio che vuole capire la teoria copernicana). 


\section{Aristotelismo e platonismo in Machiavelli}

In questa forma di aristotelismo è immerso tutto il Principe di Machiavelli. Non mancano peraltro venature platoniche e la più considerevole è forse nella catalogazione e diversificazione dei diversi tipi di principati, che sembra veramente suggerita dalla lettura dei dialoghi platonici. Sicché si direbbe che Machiavelli segua una logica platonica, ma una metodologia aristotelica. A confermarci in questa opinione c'è il fatto che tale metodologia è stata definitivamente invalidata per quei processi conoscitivi consistenti, nelle scienze sperimentali, in indagini promosse secondo strategie definibili, sperimentabili e sperimentate in base a modelli matematici. Proprio attraverso il machiavellismo, le doti intellettuali e morali del mago sono state ancora enfatizzate. Si è infatti, bene o male, continuato ad attribuirle all'uomo politico che, grazie a intuito, freddezza, intelligenza misurata delle cose e delle persone, sa quel che deve fare... Ed è inutile dire che a questo punto Machiavelli si candida ad essere postmoderno, vista la quasi coincidenza del suo principe con 1' oltre-uomo di Nietzsche. Se peraltro c'è in Nietzsche qualcosa che in Machiavelli manca del tutto, cioè la critica a una razionalità intesa quale valore normativo dei comportamenti umani, si converrà che, come dicevamo all'inizio, l'intelligenza del principe è sempre per Machiavelli un'intelligenza politica, che non si affida a un metodo, ma fiuta volta per volta la pista da seguire.

Machiavelli troppo spesso è accostato a quanti dopo di lui si sono fatti promotori di una grande e importante rivoluzione che, sul piano della vicenda culturale europea, ha portato alla nascita della moderna scienza sperimentale. La verità è che Machiavelli ha perfino un linguaggio da indovino.

I suoi distinguo, le sue catalogazioni, il suo ragionare sui remedii, i suoi consigli intorno a ciò da cui un principe deve guardarsi, sono luoghi di una retorica da oroscopo, di cui è esempio perfino la dedicatoria del Principe, là dove lo scrittore sostiene di avere " una lunga esperienza delle cose moderne e una continua lezione delle antique », " con gran diligenzia lungamente escogitate ed esaminate » («Al Magnifico Lorenzo de’ Medici », dedicatoria del Principe, § 1). Non vogliamo con questo dire che Machiavelli credesse a cose come gli oroscopi. Abbiamo già visto come l'intelligenza fosse per lui, prima ancora che capacità di seguire un ragionamento, un sapere piuttosto prevedere effetti, deducendoli da un'osservazione attenta, dove l'intuito conta più della riflessione. Ora, mentre siamo certi del guadagno derivante dall'aver liquidato l'aspetto cialtronesco e perfino burlesco di una cultura che aveva sopravvalutato l'attendibilità di certe forme di sapere, non siamo d'accordo circa l' ostinazione a trascurarne gli aspetti meno banali. Tali sono per noi quelli legati al potenziamento e all'educazione di doti personali che vanno dalle capacità mnemoniche all'affinamento dei sensi. $\mathrm{Ci}$ pare ovvio ad esempio che l'esercizio del disegno comporti una maggiore sensibilità visiva e induca chi ad esso si dedica ad osservare le cose con occhio più attento. E venendo ad arti volte ad affinare l'abilità del politico, sembra che la scherma, praticata dal principe quasi per dovere, abbia sempre avuto un ruolo nell'educazione del politico di razza. Questo ruolo le fu riconosciuto perfino nel tardo Ottocento, quando il Positivismo trionfava nelle scuole e nelle aule universitarie. 
Ora noi vediamo in Machiavelli più che il campione di una cultura che guarda allo sperimentalismo come prossimo punto d'approdo della ricerca, colui che nel dissacrare la leggenda di un sapere magico, ha sollevato di un po' il velo su certi segreti di un tale sapere. Esercizio, disciplina, applicazione ma anche coraggio, pongono l'uomo in condizione di aver ragione di un presunto cieco destino al quale per credulità popolare sarebbe legata l'esistenza degli uomini.

E' un fatto che mentre la scienza ha sempre dovuto avvolgersi nel mantello protettivo del politico, mecenate e protettore, il politico ha probabilmente finto di affidarsi alla scienza interrogando giuristi, economisti e sociologi per poi compiere i suoi passi indipendentemente dal loro giudizio, quando non abbia concesso a giuristi, economisti e sociologi compiacenti una patente di autorevolezza, conseguente a una certa loro disponibilità a giustificare le scelte già compiute dal politico.

In un celebre passo del Principe leggiamo: «E' non mi è incognito come molti hanno avuto e hanno opinione, che le cose del mondo sieno governate dalla fortuna e da Dio, che gli uomini con la prudentia loro non possino correggerle, anzi non vi abbino remedio alcuno [...] Nondimanco [...] iudico poter esser vero che la fortuna sia arbitra della metà delle azioni nostre, ma che etiam lei ne lasci governare l'altra metà, o presso, a noi...» (Il Principe, XXV, «Quanto possa la fortuna nelle cose umane e in che modo se li abbia a resistere » $\S 1$ ).

La scienza politica, dunque, non consiste nell'individuare le leggi che determinano naturalmente il fenomeno. Essa è semmai la scienza di come gli errori possano, più che correggersi, sfruttarsi per l'utile di chi li compie. In questo la scienza politica ci pare essere la scienza di domani, fondata su una sorta di epistemologia dell'errore che non escluderemmo compatibile con la postmodernità.

\section{BIBLIOGRAFIA:}

BURCKARDT Jakob (1973), L'età del rinascimento in Italia, Parte prima. Firenze, Sansoni. DE SANCTIS Francesco (1963), La giovinezza, Torino, Einaudi, p. 61.

LYOTARD Jean-François (1987), La condizione postmoderna. Rapporto sul sapere, trad. it. Milano, Feltrinelli, p. 76.

MCLUHAN Herbert Marshall, La galassia Gutenberg: la nascita dell'uomo tipografico, trad. it., Armando, Roma 1988.

Rossi Paolo (1964), Francesco Bacone. Dalla magia alla scienza, Torino, Einaudi, p. 1752.

MACHIAVElli Niccolò, La congiura de la Magione (a cura di Ludovico Fulci), Roma, Biblink, 2001, p. 35.

RUSSO Luigi (1972), Machiavelli, Bari, Laterza, p. 17.

SiCILIANo Enzo (1971), Moravia, Milano, Longanesi, p. 89.

SKINNER Quentin, Machiavelli, trad. it. Il Mulino, Bologna 1999, p. 47.

TOMMASINI Oreste (1883-1911), La vita e gli scritti di Niccolò Machiavelli nella loro relazione con il Machiavellismo, vol. II, Torino, Loescher, p. 39-41. 


\begin{abstract}
Postmodern condition involves an inquiry about modern history and its inheritance. The article is about a possible postmodernity of Machiavelli, whose thought is so interesting nowadays for many politicians and historians of culture as well. After considering some relevant aspects of Machiavelli's prince as an ideal politician (that justifies the surviving of Machiavellian and his political theories to the French Revolution), the author points out that, according to some Italian interpreters' suggestions, the Machiavellian virtus has to be considered as related to the alchemic tradition of postmedieval Italian physicians.

Although modern rationalism has invoked Machiavelli among its pioneers, it is easy to prove that the analysis of politics, as promoted by Machiavelli, is scientific in its perspective, but its approach does not ignore casualty. This is why the Machiavellian prince is so similar to the Nietzsche's Übermensch. Are such similarities so relevant to maintain that Machiavellian inheritance is important for postmodernism? A revival of the Nietzsche's philosophy seems to legitimate such an opinion and confirms that the question « is Machiavelli modern or postmodern? » does not consist in a joke. Maybe it is after a general evaluation of modernity.
\end{abstract}

